\title{
A METHOD FOR THE SEAMLINES NETWORK AUTOMATIC SELECTION BASED ON BUILDING VECTOR
}

\author{
LI Penglong ${ }^{\mathrm{ac} *}$, DONG Yichu ${ }^{\mathrm{b}}$, HU Yan ${ }^{\mathrm{a}, \mathrm{c}}$, LI Xiaolong ${ }^{\mathrm{a}, \mathrm{c}}$, TAN Pan ${ }^{\mathrm{a}, \mathrm{c}}$ \\ a. Chongqing Geomatics Centre, Chongqing China - (lpl,huyan,lxl,tp)@d1023.net \\ b. Chongqing Centre for Productivity Development, Chongqing China-(dongyichu@126.com) \\ c. Chongqing Remote Sensing Centre, Chongqing China
}

Commission III, WG III/6

KEY WORDS: Building Vector, Orthoimage Mosaicking, Seamlines Network, Automatic Selection

\begin{abstract}
:
In order to improve the efficiency of large scale orthophoto production of city, this paper presents a method for automatic selection of seamlines network in large scale orthophoto based on the buildings' vector. Firstly, a simple model of the building is built by combining building's vector, height and DEM, and the imaging area of the building on single DOM is obtained. Then, the initial Voronoi network of the measurement area is automatically generated based on the positions of the bottom of all images. Finally, the final seamlines network is obtained by optimizing all nodes and seamlines in the network automatically based on the imaging areas of the buildings. The experimental results show that the proposed method can not only get around the building seamlines network quickly, but also remain the Voronoi network' characteristics of projection distortion minimum theory, which can solve the problem of automatic selection of orthophoto seamlines network in image mosaicking effectively.
\end{abstract}

\section{INTRODUCTION}

Digital Orthophoto Map(DOM) is obtained by ortho-rectifying and mosaicking the orientated remote sensing images based on digital elevation model (DEM). Image mosaicking is the most important step to produce the orthophoto image, and the selection of the seamlines is the most important in the image mosaicking because the quality of the seamlines directly affects the quality of the orthophoto image. However, at present, the network of seamlines in most software needs to be selected and modified manually, and the degree of automation is low. So, it is of great significance to study the automatic selection method of the seamline network to improve the automation and quality of DOM. Researchers have done a great deal of research in the field of automatic selection of seamlines network, which can be mainly divided into the following three categories:

The first class of methods were based on the generalized Voronoi diagram. (Hsu S, Sawhney H S, Kumar R,2002) introduced a method to mosaic the images with the Voronoi diagram firstly. (Pan J, Wang M, et al, 2009,2010) proposed a method for automatic generation of seamline network using area Voronoi diagrams with overlap, which solved the problem that the traditional Voronoi diagram network could cause the flaw under the low overlap conditions. But these seamline networks didn't solve the problem of seamlines bypassing buildings.

The second kind of methods selected the seamlines automatically by constructing the different image of the overlapped regions between the two adjacent images. (Kerschner M,2000,2001) introduced a technique to find the best seamlines with twin-snakes operator by calculating the difference of brightness and gradient of the pixels corresponding to the overlapped region. However, the twinsnakes operator usually lost the global minimum area and could not guarantee the global optimality. (Ai H, Zhang L, Wang L, 2011) optimized the algorithm to weaken the shortcoming effectively. Dijkstra algorithm was proposed in (Davis J,1998), which was very complex and easy to lose the best path. Later, the Dijkstra algorithm was improved by (Chon J, Kim H, Lin C $\mathrm{S}, 2010$ ) with a technique minimizing the maximum local mismatch and the global cost. And (Yuan X, Zhong C,2012) improved the minimizing local maximum algorithm on searching seam line for orthoimage mosaicking and the efficiency had been improved a lot. An automatic selection method based on ant colony algorithm was proposed by (Zhang J, Sun M, Zhang Z,2009) as well as the intelligent seamlines detection algorithm based on minimum spanning tree was introduced by (Chen J, Xu B, Zhang L, et al,2015). However, the efficiency of these methods is low. Most of them are targeted to two images, and the automatic selection method for the whole mosaicking seamlines network is rarely mentioned.

Some researchers also made use of the assistant data (such as DSM, DLG, and so on) to select the mosaicking lines automatically. For example, a method was proposed by (Sun J, Ma H, Tang X,2011), which could get the areas of buildings by combing the DSM and DEM. (Zuo Z, Zhang Z, Zhang J, et al,2016) introduced a technique to detect the seamlines intelligently with retro snake algorithm based on the image segmentation. These methods didn't take the minimization of the centre projection into account. So, a method of optimizing the Voronoi network based on the building vector data to make sure the seamlines bypass the buildings is proposed, which contains following steps:

Step One: Acquisition of simple DBM (digital building model) and calculation of building's imaging area on the DOM.

Step Two: Automatic generation and simplification of the initial Voronoi network.

Step Three: Optimization of each node in the seamlines network. Step Four: Optimization of each seamline in the network.

The rest of this paper is organized as follows. The process of the new method proposed in this paper is introduced in Sect.2. Sect.3 gives the experimental results and analysis. At last, Sect. 4 is the conclusion of the method purposed in this paper.

\footnotetext{
* Corresponding author
} 


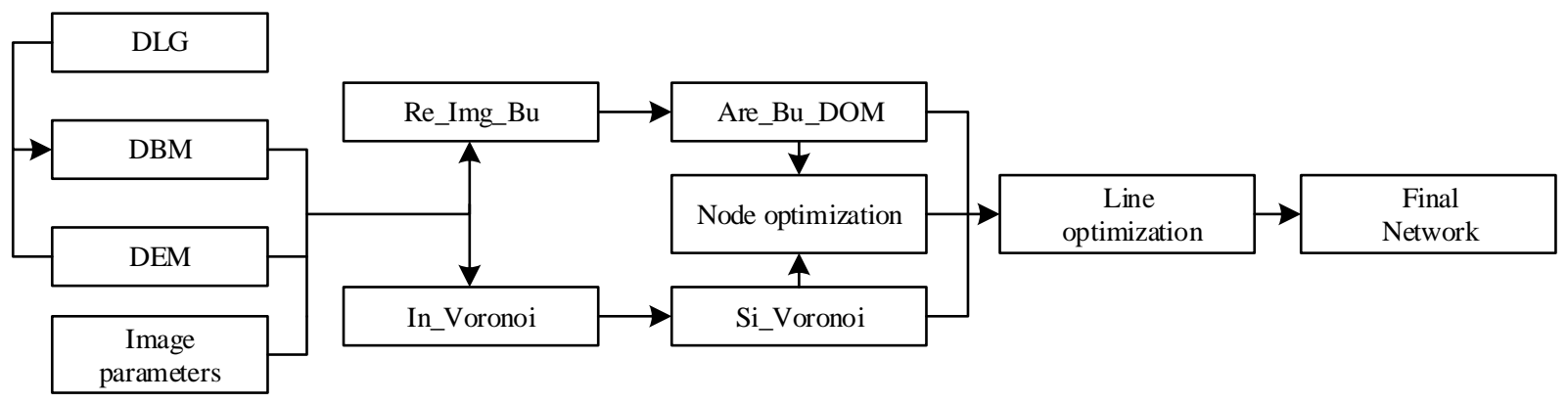

Fig.1 Flaw chart of automatic selection of the seamline network based on building vectors

\section{METHOD PROPOSED}

In order to improve the efficiency of large scale orthophoto production of city, this paper presents a method for automatic selection of seamlines network in large scale orthophoto based on the buildings' vector. Fig.1 shows the whole flaw chart of the proposed method in this paper. Firstly, a simple model of the building is built by combining building's vector, height and DEM, then the imaging area of every building on every DOM is calculated. Secondly, the initial Voronoi network of the measurement area is automatically generated and simplified based on the positions of the bottom of all images. Finally, the final seamlines network is obtained by optimizing all nodes and seamlines in the network automatically based on the imaging areas of the buildings.

\subsection{Acquisition of simple DBM and building's region on the DOM}

The planning management department carries out information collection work on urban buildings every year, which contain the height and the vector line of the buildings. So, the simple model of the building can be obtained automatically according to the height, the vector of the buildings and the DEM of the measuring area. As it shows in Fig.2, abcd is the vector polygon of the building in the DLG, and ABCD is the real roof vector which is obtained by improving the basement polygon with the height of the house. So, the simple digital model of this building ABCD-abcd is obtained.

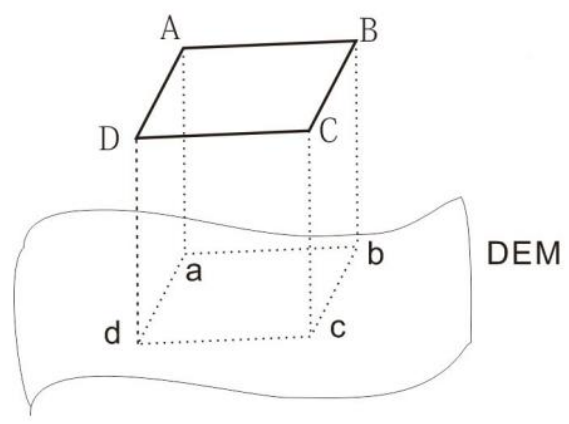

Fig.2 the acquisition of simple DBM

The imaging area of the building on the original image can be calculated according the exterior orientation elements of the original image and the coordinates of the DBM based on the formula (1). Further, the imaging area of this building on DOM can be obtained from the imaging area on original image according to formula (2) based on the method of DEM iterative (Deng F, Kang J, Li P, et al, 2015).

$$
\begin{aligned}
& \left\{\begin{array}{l}
x=-f \frac{a_{1}\left(X-X_{S}\right)+b_{1}\left(Y-Y_{S}\right)+c_{1}\left(Z-Z_{S}\right)}{a_{3}\left(X-X_{S}\right)+b_{3}\left(Y-Y_{S}\right)+c_{3}\left(Z-Z_{S}\right)} \\
y=-f \frac{a_{2}\left(X-X_{S}\right)+b_{2}\left(Y-Y_{S}\right)+c_{2}\left(Z-Z_{S}\right)}{a_{3}\left(X-X_{S}\right)+b_{3}\left(Y-Y_{S}\right)+c_{3}\left(Z-Z_{S}\right)}
\end{array}\right. \\
& \left\{\begin{array}{l}
X=X_{S}-\left(Z_{s}-Z\right) \frac{a_{1} x+a_{2} y-a_{3} f}{c_{1} x+c_{2} y-c_{3} f} \\
Y=Y_{S}-\left(Z_{s}-Z\right) \frac{b_{1} x+b_{2} y-b_{3} f}{c_{1} x+c_{2} y-c_{3} f}
\end{array}\right.
\end{aligned}
$$

Where $(\mathrm{x}, \mathrm{y})$ is the coordinate in original image

$(\mathrm{X}, \mathrm{Y}, \mathrm{Z})$ is the coordinates of the DBM's corner

(Xs, Ys,Zs) is the position of camera centre

$f$ is the focal length of the camera

$\mathrm{a}_{1} \ldots \mathrm{c}_{3}$ are parameters of the rotation matrix

As shows in Fig3, $\mathrm{O}$ is the centre's position of camera, and ABCD-abcd is the model of a building. The polygon aA'B'C'cd is the imaging area of this building on the original image and the polygon a"A"B"C"c"d" is the imaging area of this building on the orthophoto. The imaging areas of each building on each orthophoto can be obtained in the same way, then the relationships between building and each orthophoto are established, and stored in the struct named "RE_Img_Bu".

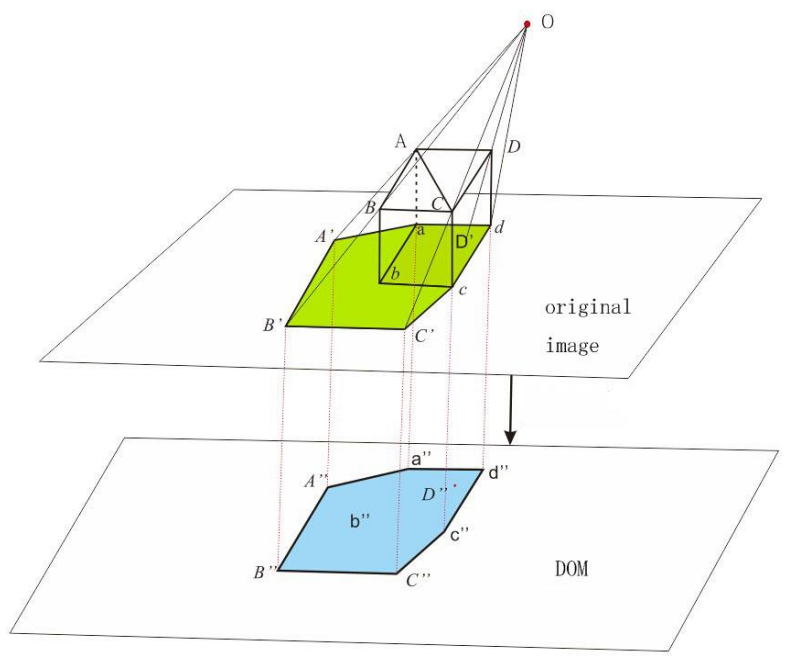

Fig.3 Acquisition of the imaging area of the building on DOM

\subsection{Generation and simplification of the Voronoi network}

The mode of the central projection and the undulation of the terrain result in the aerial images' projection distortion, and the projection distortion becomes bigger and bigger from the centre to the edges of the image. In order to make use of the minimum characteristics of projection distortion theory of Voronoi network, the initial Voronoi network is generated automatically 
base on all the central points of the aerial images (Li P, DENG $\mathrm{F}$, et al, 2018). There are many very short edges in the initial Voronoi network, as showed in Fig.4(a). In order to reduce the difficulty of network's optimization, these short edges were deleted to simplify the network firstly. And Fig.4(b) gives the simplified Voronoi network, and we can see that the characteristics of the original network are maintained. Every node connects four edges and every seamline has two nodes in the simplified Voronoi network.

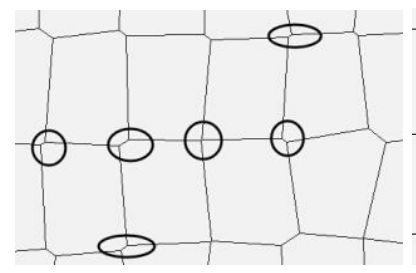

(a) Initial Voronoi

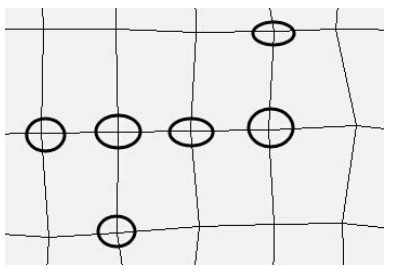

(b) Simplified Voronoi
Fig.4 Local Area of Voronoi Network

\subsection{Selection and optimization of network's nodes}

If we want the seamlines not to cross through the imaging area of the buildings, we must ensure that the nodes are not in the imaging area of the buildings. As showed in Fig.5, the same building has different imaging areas on different images. As we know, one node is related to four images, so the node must be out of the union of the imaging area of four images. For each node in the network, the optimization steps as follows:

(1) Find out all the buildings within 50 meters around this node and compute every building's union area in the related four images.

(2) Judge whether the node is in the imaging area of a building. If true, move it out, otherwise the node is a good one.

(3) Optimize the next node until all nodes are optimized.

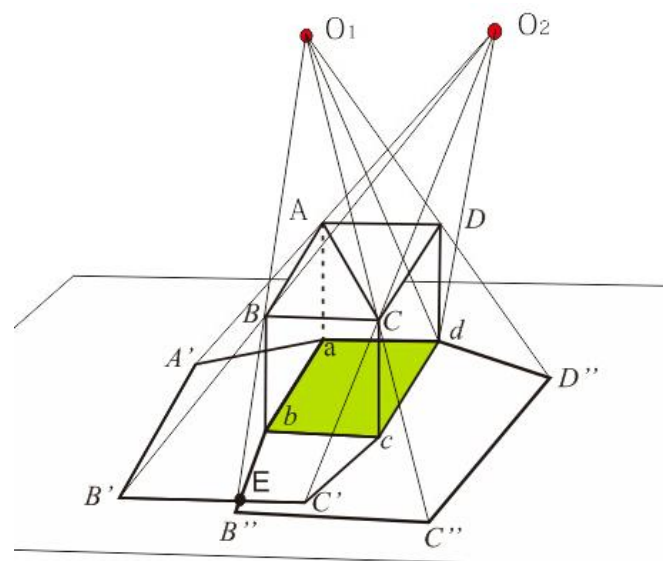

Fig.5 Imaging Areas of Buildings on Different Images

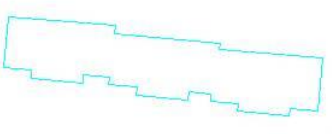

(a) building vector

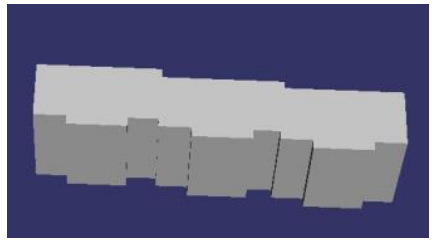

(b) simple model

\subsection{Selection and optimization of network's seamlines}

Each seamline has two nodes, and all nodes are out of the buildings' imaging area. If every seamline doesn't cross through the imaging areas of buildings, the seamline network is successful. So, for each seamline in the network, the optimization steps as follows:

(1) Find out all the buildings within 50 meters beside this seamline and compute every building's union area in the related two images.

(2) Judge whether the seamline pass through the imaging area of each building. If true, replace this part with the edges of this building imaging area polygon, until the new seamline rounds all the buildings. Otherwise, this seamline is a good one. The selection result of the seamline is shown in Fig.6.

(3) Optimize the next seamline until all seamlines are optimized.

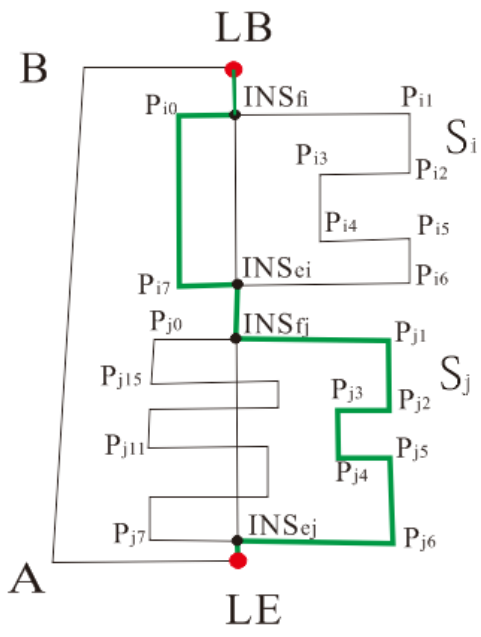

Fig.6 Optimization of Seamlines

\section{EXPERIMENTS AND DISCUSSIONS}

In order to validate the validity of this method, $32 \mathrm{UAV}$ images whose ground resolution is 0.15 meter and 526 buildings in urban are taken as the experimental data, and the experimental results are shown in Fig.7. Fig.7(a)-(d) shows the acquisition of simple DBM and building's imaging area on original image and the orthophoto. Fig.7(e)-(i) shows contrast diagram of nodes and seamlines in the network before and after the optimization. We can see that the node is moved out of the buildings' area successfully and the optimized seamlines have bypassed all the buildings. Compared Fig.7(j) and Fig.7(k), it can be seen the final DOM which mosaicked with the optimized seamlines network avoid texture misplacement. At last, the whole seamlines network before and after optimization are showed in Fig.7(l) and Fig.8(m). And we can see that the proposed method can not only get around the building seamlines network quickly, but also remain the Voronoi network' characteristics of projection distortion minimum theory.

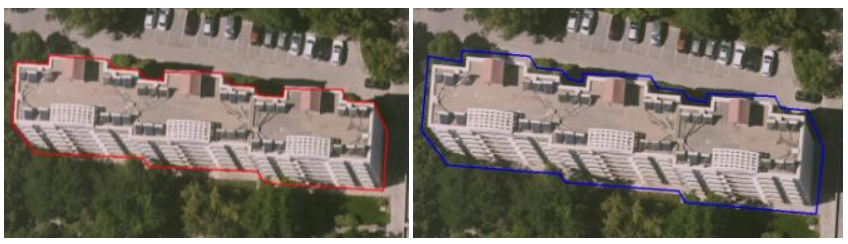

(c) imaging area in original image

(d) imaging area in orthophoto 


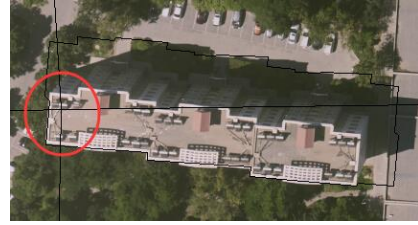

(e) original node

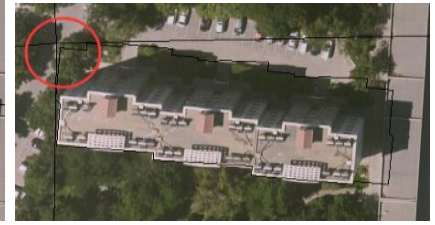

(f) optimized node

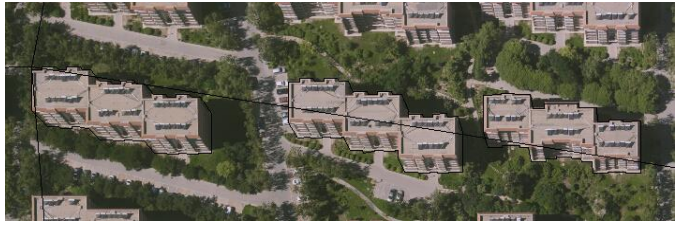

(g) original seamline

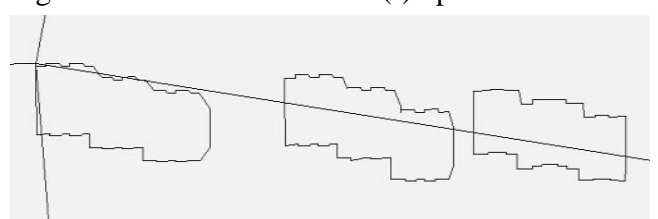

(h) original seamline

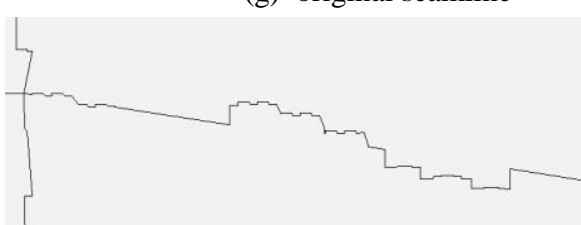

(i) optimized seamline

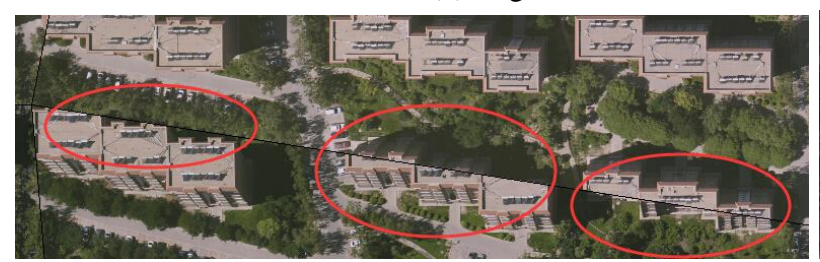

(j) original mosaicking result

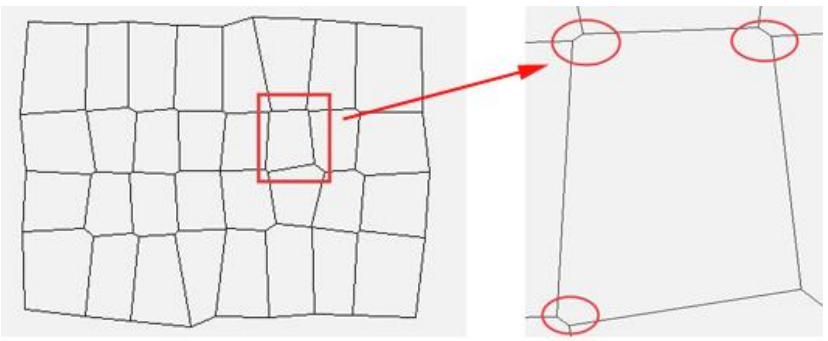

(1) initial Voronoi network

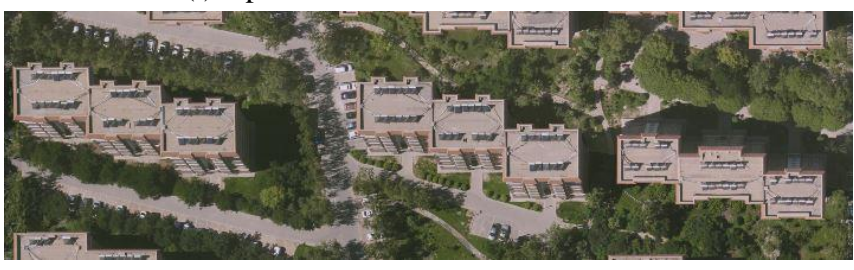

(k) final mosaicking result

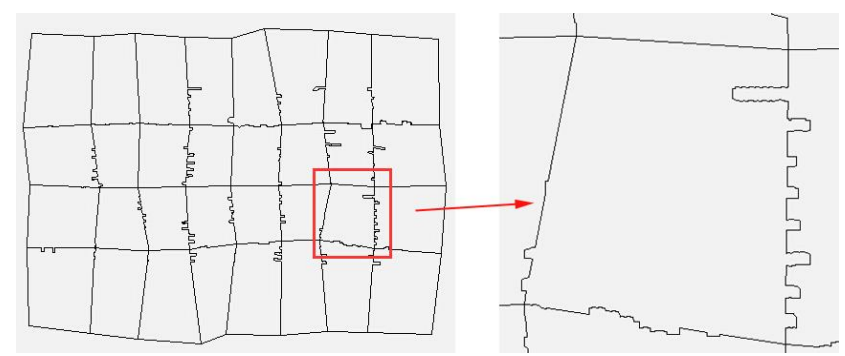

(m) final seamline network

Fig.7 Experiment Results

\section{CONCLUSION}

This paper proposes a method for the seamlines network automatic selection based on building vector to improve the efficiency of large scale orthophoto production of city. And the experimental results show that the proposed method can not only get around the building seamlines network quickly, but also remain the Voronoi network' characteristics of projection distortion minimum theory, which can solve the problem of automatic selection of orthophoto seamlines network in image mosaicking effectively.

\section{ACKNOWLEDGEMENTS}

This research was supported by Chongqing Social Undertakings and People's Livelihood Guarantee Technology Innovation (cstc2017shmsA30011).

\section{REFERENCES}

Ai H, Zhang L, Wang L, 2011. "Automatic mosaicking method for large block of orthophotos". Proc Spie, 8005(1):539-547.

Chon J, Kim H, Lin C S,2010. "Seam-line determination for image mosaicking: A technique minimizing the maximum local mismatch and the global cost." Isprs Journal of Photogrammetry \& Remote Sensing, 65(1):86-92.
Chen J, Xu B, Zhang L, et al,2015. "Fast and Intelligent Seamline Detection for Orthoimage Mosaicking Based on Minimun Spanning Tree. " Acta Geodaetica et Cartographica Sinica,44(10): 1125-1131

Davis J,1998. "Mosaics of scenes with moving objects." IEEE Computer Society Conference on Computer Vision \& Pattern Recognition. IEEE, 354-360.

Deng F, Kang J, Li P, et al,2015. "Automatic true orthophoto generation based on three-dimensional building model using multiview urban aerial images". Journal of Applied Remote Sensing, 9(1):095087-095087.

Hsu S, Sawhney H S, Kumar R,2002. "Automated Mosaics via Topology Inference". Computer Graphics \& Applications IEEE, 22(2):44-54.

Kerschner M,2000. "Twin snakes for determining seam lines in orthoimage mosaicking". International Archives of Photogrammetry \& Remote Sensing,33(4): 454-461.

Kerschner M,2001. "Seamline detection in colour orthoimage mosaicking by use of twin snakes". Isprs Journal of Photogrammetry \& Remote Sensing, 56(1):53-64.

Li P, DENG F, et al, 2018. "A method of GPU-CPU Coprocessing Rapid Images Mosaicking Based on Valid areas". Geomatics and Information Science of Wuhan University, 43(2). 
Pan J, Wang M, Li D, et al, 2009. "Automatic Generation of Seamline Network Using Area Voronoi Diagrams With Overlap ". Geomatics \& Information Science of Wuhan University, 47(6):1737-1744.

Pan J, Mi W, 2010. "Approach for Automatic Generation and Optimization of Seamline Network". Acta Geodaetica Et Cartographica Sinica, 39(3):289-294.

Sun J, Ma H, Tang X,2011. "Optimization of LIDAR System Ortho-Image Mosaic Seam-Line. " Geomatics and Information Science of Wuhan University, 36(3):325-328.

Tang J, Zhang W,2016. "A Method of Using Historical DLG Data to Optimize Seam Line in Orthophoto Mosaicing." Bulletin of Surveying and Mapping, (8):74-76.

Yuan X, Zhong C,2012. "An improvement of minimizing local maximum algorithm on searching seam line tor orthoimage mosaicking." Acta Geodaetica Et Cartographica Sinica, 41(2):199-204.

Zhang J, Sun M, Zhang Z,2009. "Automated seamline detection for orthophoto mosaicking based on ant colony algorithm. " Geomatics \& Information Science of Wuhan University, 34(6):675-678.

Zuo Z, Zhang Z, Zhang J, et al,2016. "Seamlines Intelligent Detection in Large-scale Urban Orthoimage Mosaicking. " Acta Geodaetica et Cartographica Sinica, (8):74-76. 